\title{
USING SEMANTIC RELATIONSHIPS TO ENHANCE NEURAL WORD EMBEDDINGS
}

\author{
Yanqin Yin, Xiaodong Sun, Huanhuan Lv, Pikun Wang, Hongwei Ma and Dongqiang Yang* \\ School of Computer Science and Technology, Shandong Jianzhu University, Jinan 250101, China
}

\begin{abstract}
Neural language models have significantly improved current natural language understanding tasks. However, distributional semantics, derived from neural language models is less competitive in computing semantic relatedness or similarity than other taxonomy-based methods. Although current researches seek to exploit the handcrafted semantic knowledge in ontology to improve distributional semantics, they often ignore distinguishing different functions of semantic relationships in updating or retrofitting neural word embeddings. This paper proposes retrofitting neural word embedding through semantic relationships encoded in semantic networks such as WordNet and Roget's thesaurus. We employ the hypernym/hyponym relationships to modify the asymmetric distance measure in retrofitting neural embeddings, which can fully transfer the hierarchical semantic information contained in semantic networks. In the evaluation with the gold-standard data sets, our method achieved the Spearman correlation value of 0.80, which is about $8 \%$ higher than the state-of-the-art methods in the literature.
\end{abstract}

\section{KEYWORDS}

Neural Network Word Embeddings, Semantic Relationship, Semantic Similarity

\section{INTRODUCTION}

The Neural Network Word Embeddings (NNWEs), learned from the word co-occurrence prediction, have become a cornerstone of current language technologies, and the improvement on their capacities of semantic computing can further benefit other applications such as information retrieval and word sense disambiguation. NNWEs are less capable in distinguishing semantic similarity and relatedness than other taxonomy-based methods. It may hinder downstream NLP tasks, especially in applications of dealing with lexical semantics such as word sense disambiguation and natural language understanding.

The existing methods mainly post-processed NNWEs with semantic constraints to enhance their distributional semantics (Bojanowski et al., 2017, Nguyen et al., 2016). The semantic constraints are mainly retrieved from external knowledge bases, e.g. WordNet (Miller, 1995), PPDB (Ganitkevitch \& Callison-Burch, 2014) and Roget's thesaurus (Kipfer, 2009). The post-processing methods such as Retrofitting (Faruqui et al., 2015), Counter-fitting (Mrkšić et al., 2016) and LEAR (Vulic \& Mrksic, 2018) significantly improved NNWEs in lexical semantic computation (Vulic et al., 2017a, Vulic et al., 2017b). However, while taking into account semantic transitivity contained in the hypernym/hyponym (IS-A) relationships, they updated NNWEs with identical weights on both semantically close and distant concepts that are within a certain distance in the IS-A hierarchy, which can potentially dampen the impacts of these hand-crafted semantic relationships.

Inspired by previous works on semantically post-processing and enhancing NNWEs, this paper investigates how to improve NNWEs by integrating the IS-A hierarchy into a distributional semantic space. We propose a method of direct hypernym update (DHU in short) to retrofit NNWEs. DHU employs different semantic relationships different from LEAR. LEAR imposes the same weights on the concepts within one or more links distance in the IS-A hierarchy. It will inevitably feed inaccurate semantic constraints into NNWEs, and often results in miscalculating distributional similarity.

\footnotetext{
* Correspondence author: ydq@ @djzu.edu.cn
} 
We firstly extract both synonymous and direct hypernym relationships from semantic networks, and then update NNWEs with them individually. By forcing a target word to only approach the semantically adjacent words, we can avoid the common problem of semantic incomprehension caused by cross-level use of IS-A relationships in LEAR. Experimental results show that DHU can significantly improve NNWEs in computing distributional semantics.

\section{DHU RETROFITTING}

Similar to the ATTRACT-REPEL (Mrkšić et al., 2017) and Counter-fitting (Mrkšić et al., 2016) models, we first extract semantic constraints from semantic networks, and then construct a novel retrofitting model using the direct hypernym constraints to improve NNWEs.

Let $V$ be a dictionary of word embeddings. The injected external constraints include synonym constraints $S$, antonym constraints $A$ and direct hypernym constraints $D H$. These constraints are processed as mini-batches such as $B_{S}, B_{A}$ and $B_{D H}$. In these sets, the word pair $\left(x_{l}, x_{r}\right)$ corresponds to a vector pair $\left(X_{l}, X_{r}\right)$. A mini batch of $k_{1}$ word pairs is given by $B_{S}=\left[\left(X_{l}^{1}, X_{r}^{1}\right), \cdots,\left(X_{l}^{k_{1}}, X_{r}^{k_{1}}\right)\right]$. Its negative example is $T_{S}=\left[\left(t_{l}^{1}, t_{r}^{1}\right), \cdots,\left(t_{l}^{k_{1}}, t_{r}^{k_{1}}\right)\right]$, same as similarity for $B_{A}$ and $B_{D H}$. The whole update process is as follows:

(1) Synonymy Attract:

$$
S\left(B_{S}, T_{S}\right)=\sum_{i=1}^{k_{1}}\left[\tau\left(\delta_{s y n}+\cos \left(X_{l}^{i}, t_{l}^{i}\right)-\cos \left(X_{l}^{i}, X_{r}^{i}\right)\right)+\tau\left(\delta_{s y n}+\cos \left(X_{r}^{i}, t_{r}^{i}\right)-\cos \left(X_{l}^{i}, X_{r}^{i}\right)\right)\right]
$$

$\tau(x)=\max (0, x)$ is the hinge loss function, $\delta_{\text {syn }}$ is the attract margin which determines how much closer these vectors should be to each other than to their respective negative examples.

(2) Antonymy Repel:

$$
A\left(B_{A}, T_{A}\right)=\sum_{i=1}^{k_{2}}\left[\tau\left(\delta_{a n t}+\cos \left(X_{l}^{i}, X_{r}^{i}\right)-\cos \left(X_{l}^{i}, t_{l}^{i}\right)\right)+\tau\left(\delta_{a n t}+\cos \left(X_{l}^{i}, X_{r}^{i}\right)-\cos \left(X_{r}^{i}, t_{r}^{i}\right)\right)\right]
$$

(3) Vector Space Preservation:

$$
R\left(B_{S}, B_{A}\right)=\sum_{X_{i} \in V\left(B_{S} \cup B_{A}\right)} \lambda_{\text {reg }}\left\|X_{i}-X_{i}\right\|_{2}
$$

where $\lambda_{\text {reg }}$ is the $L 2$ regulation constant and $\hat{X}_{i}$ denotes the original word vector for word $x_{i}$.

(4) Direct Hypernym Update

$$
D H\left(B_{D H}\right)=\sum_{i=1}^{k_{3}} \frac{|x|-|y|}{|x|+|y|}
$$

The full cost function is given by the sum of above the four formulas:

$$
C=S\left(B_{S}, T_{S}\right)+A\left(B_{A}, T_{A}\right)+D H\left(B_{D H}\right)+R\left(B_{S}, B_{A}, B_{D H}\right)
$$

\section{EXPERIMENT SETUP}

\subsection{NNWEs}

We tested DHU with three widely used NNWEs:

1) GloVe Common Crawl-300D ${ }^{1}$ (GloVe)

2) GoogleNews-Vectors-Negative300-SLIM² (W2V-SLIM)

3) Paragram $^{3}(300 \mathrm{D})$

\footnotetext{
1 https://nlp.stanford.edu/projects/glove/

2 https://github.com/eyaler/word2vec-slim

3 https://www.cs.cmu.edu/ jwieting/
} 


\subsection{Semantic Constraints}

The synonymous, antonymous, and direct hypernym constraints were extracted from the two well-known semantic resources: WordNet and Roget's thesaurus. Among them, the synonymous constraints consist of 909,302 pairs and 554,507 pairs of synonyms, respectively; the antonyms are 465,406 pairs and 39,283 pairs. The direct hypernym constraints were taken only from WordNet, having a total of 320,586 pairs.

\subsection{Hyper-parameters Optimization}

To speed up the training process, we used AdaGrad (Duchi et al., 2011) to iteratively train the NNWEs. We set the initial learning rate to 0.05 . The experimental hyper-parameters were optimized through grid search, i.e. $\quad \lambda_{\text {reg }} \in\left[10^{-3}, 10^{-4}, \cdots, 10^{-10}\right], \delta_{\text {syn }}, \delta_{\text {ant }} \in[0,0.1, \cdots, 0.9,1]$, and $k_{1}, k_{2}, k_{3} \in[32,64,128,256]$. By fine-tuning on the gold-standard data set of WordSim-353-similarity (Agirre et al., 2009), we achieved the optimal hyper-parameter values, i.e. $\lambda_{\text {reg }}=10^{-9}, \delta_{\text {syn }}=0.6, \delta_{\text {ant }}=0.0$, and $k_{1}, k_{2}, k_{3}=128$.

\subsection{Results and Analysis}

To evaluate DHU on retrofitting NNWEs, we used the public data sets of SimLex-999 (Hill et al., 2015) and SimVerb-3500 (Gerz et al., 2016) as the tests sets. The Spearman rank correlation coefficient was calculated to show how close NNWEs can approach in comparison with human similarity ratings.

Figure 1 summarized retrofitting results of GloVe, W2V-SLIM, and Paragram on the above test sets. The retrofitting methods, in turn, are Retrofitting, Counter-fitting, LEAR, and DHU.

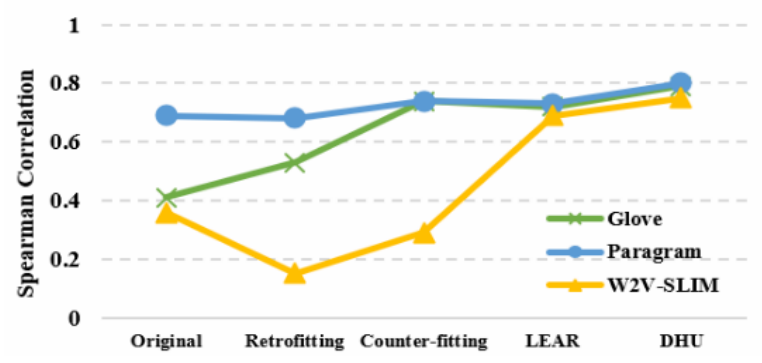

(a)

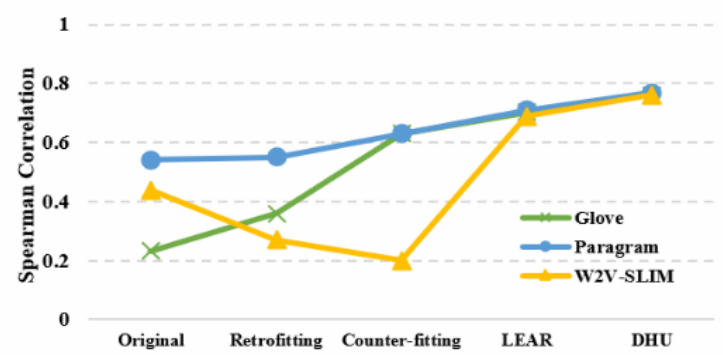

(b)

Figure 1. The performance of retrofitting word embeddings (a) SimLex-999 and (b) SimVerb-3500

The Retrofitting method significantly improved GloVe, but it could not work on Paragram and W2V-SLIM. It suggested that since Paragram was semantically initialized, and W2V-SLIM filters out synonymous concepts, which could restrict the retrofitting effects. The Counter-fitting results indicated that injecting both synonymous and antonymous constraints can significantly improve semantic similarity prediction.

Compared with Counter-fitting, LEAR achieved a relatively better result, which may indicate that injecting the IS-A constraints may be beneficial to enhance NNWEs. However, LEAR also introduced the IS-A hierarchical relationships without explicitly distinguishing their semantic differences in updating NNWEs, which may be detrimental to Paragram.

Our DHU method greatly improved the three NNWEs, partly because semantic distance under the direct hypernyms are relatively short, which are more reliable to function as semantic constraints; partly because DHU can take into account both the asymmetry of semantic relationship and directionality of semantic transferability between hypernyms and hyponyms (Marathe \& Hirst, 2010). Given the increase of path length, concept similarity may decrease significantly while traversing along IS-A relationships. The hypernym constraints in DHU were retrieved from their directly adjacent words in WordNet, which may be beneficial to updating NNWEs. The experimental results also show that DHU can reduce uncertainty caused by the multi-level semantic transferability issues in LEAR.

To analyze semantic representations after retrofitting NNWEs, we adopted the TSNE method (Laurens \& Hinton, 2008) to visualize GloVe. 
In Figure 2, the similar words represented in GloVe are relatively sparse in the reduced 2-dimensional space. After retrofitting by LEAR, although the distance between cat and cats is shortened, the distance between cat and its synonyms was pushed further away. After the DHU retrofitting, bobcat along with cat was pulled closer than them in the LEAR retrofitting. DHU also added cheetah, leopard, panther and lynx to the top 5 distributional similar words of cat. These words belong to feline and have direct hypernym relationship with cat. They are semantically closer to cat. The semantic space after the DHU retrofitting, shown in Figure 2-(c), is more compact, which highlights the effectiveness of DHU.

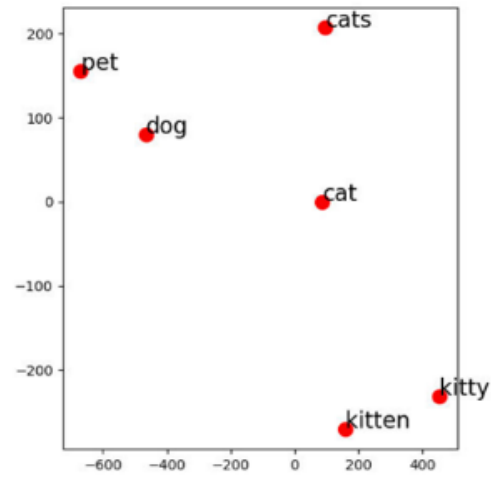

(a)

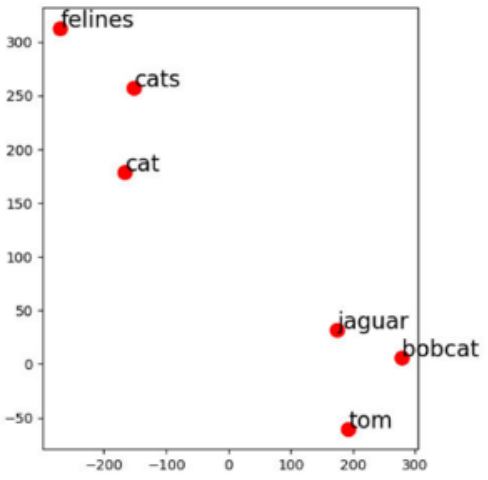

(b)

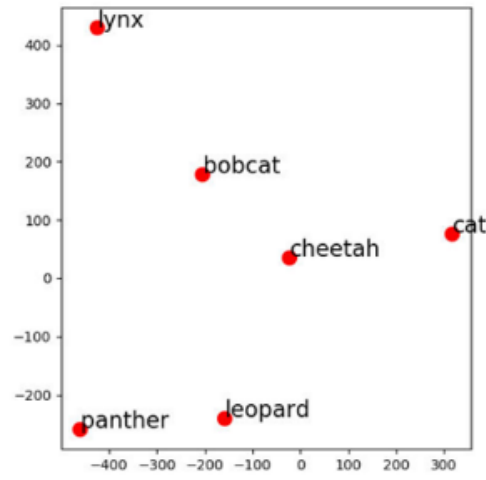

(c)

Figure 2. Demo of word positions in a 2-D space. We show the word cat and its top 5 distributional similar words in different neural embeddings an example to show the top 5 words are similar to cat.

(a) Original GloVe (b) GloVe after the LEAR retrofitting (c) GloVe after the DHU retrofitting

\section{CONCLUSION}

This paper proposed a novel retrofitting method of DHU to enhance NNWEs. Through injecting the direct hypernym constraints into NNWEs, we addressed the semantics disappearance after a multi-level traverse of IS-A relationships. DHU can effectively merge semantic knowledge into NNWEs. In the future study, we will seek different ways of incorporating other semantic relationships such as meronym-holonym (HAS-A) into NNWEs.

\section{ACKNOWLEDGEMENT}

This research was supported by the National Social Science Foundation of China (Grant No. 17BYY119).

\section{REFERENCES}

Agirre, E., Alfonseca, E., et al., 2009. A Study on Similarity and Relatedness Using Distributional and WordNet-based Approaches. Proceedings of NAACL. Boulder, Colorado, Association for Computational Linguistics, 19-27.

Bojanowski, P., Grave, E., et al., 2017. Enriching word vectors with subword information. In Transactions of the Association for Computational Lingus. 5, 135-146.

Duchi, J. C., Hazan, E., et al., 2011. Adaptive Subgradient Methods for Online Learning and Stochastic Optimization. Journal of Machine Learning Research. 12, 7, 2121-2159.

Faruqui, M., Dodge, J., et al., 2015. Retrofitting word vectors to semantic lexicons. Proceddings of NAACL. Denver, Colorado, Association for Computational Linguistics, 1606-1615.

Ganitkevitch, J. and Callison-Burch, C., 2014. The Multilingual Paraphrase Database. Proceddings of the Ninth International Conference on Language Resources and Evaluation. Reykjavik, Iceland, European Language Resources Association, 4276-4283.

Gerz, D., Vulic, I., et al., 2016. SimVerb-3500: A Large-Scale Evaluation Set of Verb Similarity. Proceddings of EMNLP. Austin, Texas, Association for Computational Linguistics, 2173-2182. 
Hill, F., Reichart, R., et al., 2015. Simlex-999: Evaluating semantic models with genuine similarity estimation. arXiv: Computational Linguistics. 41, 4, 665-695.

Kipfer, B. A., 2009. Roget's 21st Century Thesaurus. Random House US, US.

Laurens, V. D. M. and Hinton, G., 2008. Visualizing Data using t-SNE. Journal of Machine Learning Research. 9, 2605, 2579-2605.

Marathe, M. and Hirst, G., 2010. Lexical Chains Using Distributional Measures of Concept Distance. International Conference on Intelligent Text Processing and Computational Linguistics. Berlin, Heidelberg, Springer, 291-302.

Miller, G. A., 1995. WordNet: a lexical database for English. Communications of the ACM. 38, 11, 39-41.

Mrkšić, N., Séaghdha, D. O., et al., 2016. Counter-fitting word vectors to linguistic constraints. arXiv preprint arXiv: 1630.00892

Mrkšić, N., Vulić, I., et al., 2017. Semantic Specialization of Distributional Word Vector Spaces using Monolingual and Cross-Lingual Constraints. In Transaction of Association for Computational Lingusitics. 5, 8, 309-324.

Nguyen, K. A., Walde, S. S. I., et al., 2016. Integrating distributional lexical contrast into word embeddings for antonymsynonym distinction. Proceddings of the 54th Annual Meeting of the Association for Computational Linguistics. Association for Computational Linguistics, 454-459.

Vulic, I. and Mrksic, N., 2018. Specialising Word Vectors for Lexical Entailment. Proceddings of NAACL. New Orleans, Louisiana, Association for Computational Linguistics, 1134-1145.

Vulic, I., Mrksic, N., et al., 2017a. Cross-Lingual Induction and Transfer of Verb Classes Based on Word Vector Space Specialisation. Proceddings of EMNLP. Copenhagen, Denmark, Association for Computational Linguistics, 2546-2558.

Vulic, I., Mrksic, N., et al., 2017b. Morph-fitting: Fine-tuning word vector spaces with simple language-specific rules. Proceedings of the 55th Annual Meeting of the Association for Computational Linguistics Vancouver, Canada, Association for Computational Linguistics, 56-68. 